\title{
EXTENSIONS OF MODULES OVER POLYNOMIAL RINGS
}

\section{MOHAMMED ELHASSANI CHARKANI and AHMED NAJIM}

Department of Mathematics

Faculty of Science Dhar Mahraz

Sidi Mohamed Ben Abdellah University

P. O. Box 1796 Atlas

$30000 \mathrm{Fez}$

Morocco

e-mail: mohammed.charkanielhassani@usmba.ac.ma ahmed.najim@usmba.ac.ma

\begin{abstract}
For a commutative and unitary ring $R$, we characterize the classes of a classical equivalence relation defined on extensions of an $R[X]$-module, which is $R$-projective by another $R[X]$-module. The modules that are extensions of an $R[X]$-module $M_{2}$, which is $R$-projective by an $R[X]$-module $M_{1}$ have a fairly simple form which we denote by $M_{1} \times_{f} M_{2}$, where $f$ is an $R$-homomorphism from $M_{2}$ to $M_{1}$. For $R, M_{1}, M_{2}$ and $f$ as in the foregoing, but $M_{2}$ is not necessarily $R$-projective, we search to classify the $R[X]$-modules $M_{1} \times_{f} M_{2}$.
\end{abstract}

2010 Mathematics Subject Classification: 13B25, $13 \mathrm{C} 05$.

Keywords and phrases: extension of modules, globally isomorphic modules, polynomial ring, strongly similar matrices.

Received July 31, 2015

(C) 2015 Scientific Advances Publishers 


\section{Introduction}

All rings considered in this paper are supposed to be with unit. Let $R$ be a commutative ring. Let $N$ and $L$ be two $R$-modules. We recall (see $[1,7,9])$ that an extension of $L$ by $N$ is a triple $(u, M, v)$, where $M$ is an $R$-module and $u, v$ are $R$-module homomorphisms such that the sequence

$$
O \rightarrow N \stackrel{u}{\rightarrow} M \stackrel{v}{\rightarrow} L \rightarrow O
$$

is exact. Two extensions $(u, M, v)$ and $\left(u^{\prime}, M^{\prime}, v^{\prime}\right)$ of $L$ by $N$ are equivalent if there exists an isomorphism of $R$-modules $\varphi: M \rightarrow M^{\prime}$ such that the following diagram is commutative:

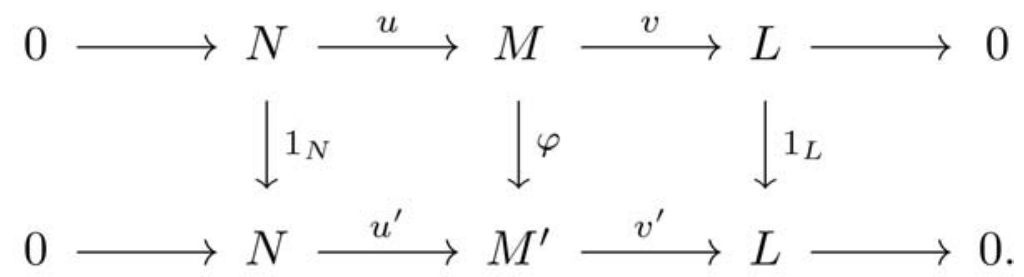

For this equivalence relation, the class of an extension $(u, M, v)$ of $L$ by $N$ is denoted by $\overline{(u, M, v)}$.

On the group rings, one of the technics used to study the module extensions consists to show that a module, which is extension of a module $L$ by another module $N$ - under some conditions - can be written in a simple form that is denoted by $N \times_{\alpha} L$, where $\alpha$ is a cocycle or a derivation (see, for example, [2], [3], [8]). For the polynomial rings, this form is much simpler, indeed instead of cocycles or derivations we can take homomorphisms of $R$-modules.

The polynomial ring with coefficients in $R$ will be denoted by $R[X]$.

In this paper, we study the extensions of $R[X]$-modules. We search essentially to characterize these extensions. In Section 2, we characterize the classes of this equivalence relation defined on extensions of an $R[X]$-modules $M_{2}$, which is $R$-projective by an $R[X]$-module $M_{1}$. As we 
already said, the modules that are extensions of an $R[X]$-module $M_{2}$, which is $R$-projective by an $R[X]$-modules $M_{1}$ have a fairly simple form which we denote by $M_{1} \times_{f} M_{2}$, where $f$ is an $R$-homomorphism from $M_{2}$ to $M_{1}$. In Section 3, for $M_{1}, M_{2}$ and $f$ as in the foregoing, but $M_{2}$ is not necessarily $R$-projective, we search to classify the $R[X]$-modules $M_{1} \times_{f} M_{2}$. In the same time, we give some properties of these modules.

\section{Extension Equivalence of Modules Over Polynomial Rings}

Let $R$ be a commutative ring. Let $M_{1}$ and $M_{2}$ be two $R[X]$-modules. Let $f \in \operatorname{Hom}_{R}\left(M_{2}, M_{1}\right)$, where $\operatorname{Hom}_{R}\left(M_{2}, M_{1}\right)$ is the set of all $R$-module morphisms from the $R$-module $M_{2}$ to the $R$-module $M_{1}$. Then, we can define a structure of $R[X]$-module on $M_{1} \times M_{2}$ by $\forall\left(m_{1}, m_{2}\right) \in$ $M_{1} \times M_{2}, X .\left(m_{1}, m_{2}\right)=\left(X . m_{1}+f\left(m_{2}\right), X . m_{2}\right)$. The module $M_{1} \times M_{2}$ equipped with this structure of $R[X]$-module will be denoted by $M_{1} \times_{f} M_{2}$. In this case, the following sequence $O \rightarrow M_{1} \stackrel{i_{1}}{\rightarrow} M_{1} \times_{f} M_{2}$ $\stackrel{p_{2}}{\rightarrow} M_{2} \rightarrow O$, is an exact sequence of $R[X]$-modules, where $i_{1}$ is the first injection and $p_{2}$ is the second projection.

Theorem 2.1. Let $O \rightarrow M_{1} \stackrel{u}{\rightarrow} M \stackrel{v}{\rightarrow} M_{2} \rightarrow O$ be an exact sequence of $R[X]$-modules. If $M_{2}$ is R-projective, then there exists $f \in \operatorname{Hom}_{R}\left(M_{2}, M_{1}\right)$ such that $\overline{(u, M, v)}=\overline{\left(i_{1}, M_{1} \times_{f} M_{2}, p_{2}\right)}$.

Proof. $O \rightarrow M_{1} \stackrel{u}{\rightarrow} M \stackrel{v}{\rightarrow} M_{2} \rightarrow O$ is exact and $M_{2}$ is projective as $R$-module. Then, there exist $v^{\prime} \in \operatorname{Hom}_{R}\left(M_{2}, M\right)$ and $u^{\prime} \in \operatorname{Hom}_{R}\left(M, M_{1}\right)$ such that $u^{\prime} o u=i d_{M_{1}}$ and $v o v^{\prime}=i d_{M_{2}}$. It is well-known that $\theta: M \rightarrow M_{1} \oplus M_{2}$ defined by $m \mapsto\left(u^{\prime}(m), v(m)\right)$ is an isomorphism of 
$R$-modules, and $\theta^{-1}: M_{1} \oplus M_{2} \rightarrow M$ is defined by $\left(m_{1}, m_{2}\right) \mapsto m+u(x)$, where $m$ is such that $v(m)=m_{2}$ and $x \in M_{1}$ is such that $x=m_{1}-u^{\prime}(m)$. We define a structure of $R[X]$-module on $M_{1} \oplus M_{2}$ by

$$
\forall\left(m_{1}, m_{2}\right) \in M_{1} \oplus M_{2}, X .\left(m_{1}, m_{2}\right)=\theta\left(X . \theta^{-1}\left(\left(m_{1}, m_{2}\right)\right)\right) .
$$

Then,

$$
\begin{aligned}
X .\left(m_{1}, m_{2}\right) & =\theta\left(X . \theta^{-1}\left(\left(m_{1}, m_{2}\right)\right)\right) \\
& =\theta(X .(m+u(x))) \\
& =\theta(X . m+X . u(x)) \\
& =\left(u^{\prime}(X . m+X . u(x)), v(X . m+X . u(x))\right) \\
& =\left(u^{\prime}(X . m)+X . x, X . m_{2}\right) \\
& =\left(X . m_{1}+u^{\prime}(X . m)-X . u^{\prime}(m), X . m_{2}\right) .
\end{aligned}
$$

We have $v(m)=m_{2}$, so there exists $m^{\prime} \in \operatorname{ker}(v)$ such that $v^{\prime}\left(m_{2}\right)=$ $m+m^{\prime}$. As $\operatorname{ker}(v)=\operatorname{Im}(u)$, then there exists $m_{2}^{\prime} \in M_{1}$ such that $v^{\prime}\left(m_{2}\right)=m+u\left(m_{2}^{\prime}\right)$. So,

$$
\begin{aligned}
u^{\prime}(X . m)-X . u^{\prime}(m) & =u^{\prime}\left(X .\left(v^{\prime}\left(m_{2}\right)-u\left(m_{2}^{\prime}\right)\right)\right)-X . u^{\prime}\left(v^{\prime}\left(m_{2}\right)-u\left(m_{2}^{\prime}\right)\right) \\
= & u^{\prime}\left(X . v^{\prime}\left(m_{2}\right)\right)-u^{\prime}\left(X . u\left(m_{2}^{\prime}\right)\right)-X .\left(u^{\prime} o v^{\prime}\left(m_{2}\right)\right)+X .\left(u^{\prime} o u\left(m_{2}^{\prime}\right)\right) \\
= & u^{\prime}\left(X . v^{\prime}\left(m_{2}\right)\right)-X .\left(u^{\prime} o v^{\prime}\left(m_{2}\right)\right) .
\end{aligned}
$$

Therefore,

$$
\begin{aligned}
X .\left(m_{1}, m_{2}\right) & =\left(X . m_{1}+u^{\prime}\left(X . v^{\prime}\left(m_{2}\right)\right)-X .\left(u^{\prime} o v^{\prime}\left(m_{2}\right)\right), X . m_{2}\right) \\
& =\left(X . m_{1}+f\left(m_{2}\right), X . m_{2}\right),
\end{aligned}
$$

where $f \in \operatorname{Hom}_{R}\left(M_{2}, M_{1}\right)$ is defined by

$$
\forall m_{2} \in M_{2}, \quad f\left(m_{2}\right)=u^{\prime}\left(X . v^{\prime}\left(m_{2}\right)\right)-X .\left(u^{\prime} o v^{\prime}\left(m_{2}\right)\right) .
$$

$\theta: M \rightarrow M_{1} \times_{f} M_{2}$ is an isomorphism of $R[X]$-modules and we have $\theta o u=i_{1}$ and $p_{2} o \theta=v$. 
Theorem 2.2. Let $f, g \in \operatorname{Hom}_{R}\left(M_{2}, M_{1}\right)$, where $M_{1}$ and $M_{2}$ are two $R[X]$-modules. Then, the following conditions are equivalent:

(1) $\overline{\left(i_{1}, M_{1} \times_{f} M_{2}, p_{2}\right)}=\overline{\left(i_{1}, M_{1} \times_{g} M_{2}, p_{2}\right)}$.

(2) There exists $h \in \operatorname{Hom}_{R}\left(M_{2}, M_{1}\right)$ such that, for all $m_{2} \in M_{2}$,

$$
f\left(m_{2}\right)+h\left(X . m_{2}\right)=X . h\left(m_{2}\right)+g\left(m_{2}\right) .
$$

Proof. (1) $\Rightarrow$ (2): If $\overline{\left(i_{1}, M_{1} \times_{f} M_{2}, p_{2}\right)}=\overline{\left(i_{1}, M_{1} \times_{g} M_{2}, p_{2}\right)}$, then there exists a homomorphism of $R[X]$-modules $\varphi: M_{1} \times_{f} M_{2} \rightarrow M_{1} \times_{g} M_{2}$ such that $\varphi \circ i_{1}=i_{1}$ and $p_{2} o \varphi=p_{2}$. Let $\left(m_{1}, m_{2}\right) \in M_{1} \times M_{2}$. We have

$$
\begin{aligned}
\varphi\left(\left(m_{1}, 0\right)\right) & =\varphi \dot{p o i}_{1}\left(m_{1}\right) \\
& =i_{1}\left(m_{1}\right) \\
& =\left(m_{1}, 0\right) .
\end{aligned}
$$

We put $\varphi\left(\left(0, m_{2}\right)\right)=\left(m_{1}^{\prime}, m_{2}^{\prime}\right)$. As $p_{2} o \varphi=p_{2}$, then $m_{2}^{\prime}=m_{2}$. We define an application $h: M_{2} \rightarrow M_{1}$ by $h\left(m_{2}\right)=m_{1}^{\prime}$. Then, we have $\varphi\left(\left(m_{1}, m_{2}\right)\right)=\left(m_{1}+h\left(m_{2}\right), m_{2}\right)$. We first show that $h$ is a homomorphism of $R$-modules. Let $m_{2}, m_{2}^{\prime} \in M_{2}$ and let $r \in R$. $\varphi\left(\left(0, m_{2}+m_{2}^{\prime}\right)\right)$ $=\left(h\left(m_{2}+m_{2}^{\prime}\right), m_{2}+m_{2}^{\prime}\right)$. But,

$$
\begin{aligned}
\varphi\left(\left(0, m_{2}+m_{2}^{\prime}\right)\right) & =\varphi\left(\left(0, m_{2}\right)\right)+\varphi\left(\left(0, m_{2}^{\prime}\right)\right) \\
& =\left(h\left(m_{2}\right), m_{2}\right)+\left(h\left(m_{2}^{\prime}\right), m_{2}^{\prime}\right) \\
& =\left(h\left(m_{2}\right)+h\left(m_{2}^{\prime}\right), m_{2}+m_{2}^{\prime}\right) .
\end{aligned}
$$

So, $h\left(m_{2}+m_{2}^{\prime}\right)=h\left(m_{2}\right)+h\left(m_{2}^{\prime}\right)$. We have also $\varphi\left(\left(0, r . m_{2}\right)\right)=\left(h\left(r . m_{2}\right), r . m_{2}\right)$. But,

$$
\begin{aligned}
\varphi\left(\left(0, r . m_{2}\right)\right) & =\varphi\left(r\left(0, m_{2}\right)\right) \\
& =r \varphi\left(\left(0, m_{2}\right)\right) \\
& =r\left(h\left(m_{2}\right), m_{2}\right) \\
& =\left(r . h\left(m_{2}\right), r . m_{2}\right) .
\end{aligned}
$$


So, $h\left(r . m_{2}\right)=r . h\left(m_{2}\right)$. Therefore, $h \in \operatorname{Hom}_{R}\left(M_{2}, M_{1}\right)$. Let $m_{2} \in M_{2}$. $\varphi\left(X .\left(0, m_{2}\right)\right)=X . \varphi\left(\left(0, m_{2}\right)\right)$. But,

$$
\begin{aligned}
\varphi\left(X .\left(0, m_{2}\right)\right) & \left.=\varphi\left(f\left(m_{2}\right), X . m_{2}\right)\right) \\
& =\left(f\left(m_{2}\right)+h\left(X . m_{2}\right), X . m_{2}\right),
\end{aligned}
$$

and

$$
\begin{aligned}
X . \varphi\left(\left(0, m_{2}\right)\right) & =X .\left(h\left(m_{2}\right), m_{2}\right) \\
& =\left(X . h\left(m_{2}\right) \cdot g\left(m_{2}\right), X . m_{2}\right) .
\end{aligned}
$$

Therefore, $\forall m_{2} \in M_{2}, f\left(m_{2}\right)+h\left(X . m_{2}\right)=X . h\left(m_{2}\right)+g\left(m_{2}\right)$.

$(2) \Rightarrow(1)$ : Assume that there exists $h \in \operatorname{Hom}_{R}\left(M_{2}, M_{1}\right)$ such that, $\forall m_{2} \in M_{2}, f\left(m_{2}\right)+h\left(X . m_{2}\right)=X . h\left(m_{2}\right)+g\left(m_{2}\right)$. We define an application $\varphi: M_{1} \times_{f} M_{2} \rightarrow M_{1} \times_{g} M_{2} \quad$ by $\forall\left(m_{1}, m_{2}\right) \in M_{1} \times_{f} M_{2}, \varphi\left(\left(m_{1}, m_{2}\right)\right)=$ $\left(m_{1}+h\left(m_{2}\right), m_{2}\right)$. We easily see that $\varphi$ is a homomorphism of $R$-modules. Let $\left(m_{1}, m_{2}\right) \in M_{1} \times_{f} M_{2}$.

$$
\begin{aligned}
\varphi\left(X .\left(m_{1}, m_{2}\right)\right) & =\varphi\left(\left(X . m_{1}+f\left(m_{2}\right), X . m_{2}\right)\right) \\
& =\left(X . m_{1}+f\left(m_{2}\right)+h\left(X . m_{2}\right), X . m_{2}\right) \\
& =\left(X . m_{1}+X . h\left(m_{2}\right)+g\left(m_{2}\right), X . m_{2}\right) \\
& =\left(X .\left(m_{1}+h\left(m_{2}\right)\right)+g\left(m_{2}\right), X . m_{2}\right) \\
& =X .\left(m_{1}+h\left(m_{2}\right), m_{2}\right) \\
& =X . \varphi\left(\left(m_{1}, m_{2}\right)\right) .
\end{aligned}
$$

Therefore, $\varphi$ is a homomorphism of $R[X]$-modules, and it is easy to see that $\varphi o i_{1}=i_{1}$ and $p_{2} o \varphi=p_{2}$. 
Remark 2.3. If we assume that the conditions of Theorem 2.2 are satisfied, then we have seen in the proof of this theorem, that the homomorphism $\varphi$ such that the following diagram of $R[X]$-modules is commutative:

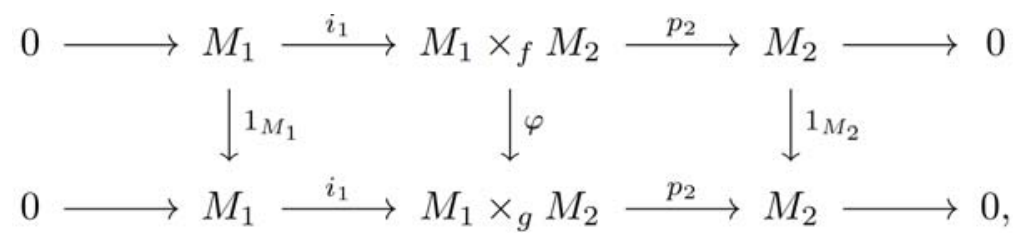

is defined by $\forall\left(m_{1}, m_{2}\right) \in M_{1} \times_{f} M_{2}, \varphi\left(\left(m_{1}, m_{2}\right)\right)=\left(m_{1}+h\left(m_{2}\right), m_{2}\right)$. (Here, we kept the notation of Theorem 2.2.) $\varphi$ is necessarily an isomorphism of $R[X]$-modules and $\varphi^{-1}$ is defined by $\forall\left(m_{1}, m_{2}\right) \in M_{1} \times_{g} M_{2}$, $\varphi^{-1}\left(\left(m_{1}, m_{2}\right)\right)=\left(m_{1}-h\left(m_{2}\right), m_{2}\right)$.

Let $M_{1}$ and $M_{2}$ be two $R[X]$-modules that are $R$-free of finite rank. Let $\mathbf{B}_{1}$ and $\mathbf{B}_{2}$ be two bases of $M_{1}$ and $M_{2}$, respectively. Let $C_{f}=\operatorname{Mat}_{\mathbf{B}_{2}, \mathbf{B}_{1}}(f)$ and $C_{g}=\operatorname{Mat}_{\mathbf{B}_{2}, \mathbf{B}_{1}}(g)$ be the matrices of $f$ and $g$, respectively, with respect to the bases $\mathbf{B}_{2}$ and $\mathbf{B}_{1}$, and let $A$ (respectively, $B$ ) be the matrix representing the action of $X$ on $M_{1}$ with respect to the base $\mathbf{B}_{1}$ (respectively, $M_{2}$ with respect to the base $\mathbf{B}_{2}$ ).

Corollary 2.4. The following conditions are equivalent:

(1) $\overline{\left(i_{1}, M_{1} \times_{f} M_{2}, p_{2}\right)}=\overline{\left(i_{1}, M_{1} \times_{g} M_{2}, p_{2}\right)}$.

(2) There exists $H \in M_{n_{1}, n_{2}}(R)$ such that, $A H-H B=C_{f}-C_{g}$.

Proof. Just take $H=M_{a t} t_{\mathbf{B}_{2}, \mathbf{B}_{1}}(h)$.

In the rest of this section, we give some interesting results on the $R[X]$-modules $M_{1} \times_{f} M_{2}$, where we use the following notations:

$$
\operatorname{Ker}(f)=\left\{x \in M_{1} \mid f(x)=0\right\} \text { and } \operatorname{Im}(f)=\left\{f(x) \mid x \in M_{1}\right\} \text {. }
$$


Proposition 2.5. The following assertions are true:

(1) Each direct summand of $M_{2}$ included in $\operatorname{Ker}(f)$ is a direct summand of $M_{1} \times_{f} M_{2}$.

(2) If $N_{1}$ is a direct summand of $M_{1}$ and $\operatorname{Im}(f) \subseteq N_{1}$, then $N_{1} \times_{f} M_{2}$ is a direct summand of $M_{1} \times_{f} M_{2}$.

Proof. (1) If $M_{2}=M_{2}^{\prime} \oplus M_{2}^{\prime \prime}$, where $M_{2}^{\prime}$ and $M_{2}^{\prime \prime}$ are two $R[X]$-submodules of $M_{2}$ such that $M_{2}^{\prime} \subset \operatorname{Ker}(f)$, then $M_{1} \times_{f} M_{2}=M_{1} \times_{f}$ $M_{2}^{\prime \prime} \oplus\{0\} \times_{f} M_{2}^{\prime}$.

(2) If $N_{1}$ is an $R[X]$-submodule of $M_{1}$ such that $\operatorname{Im}(f) \subseteq N_{1}$, and there exists an $R[X]$-submodule of $M_{1}$ such that $M_{1}=N_{1} \oplus N_{1}^{\prime}$, then $M_{1} \times_{f} M_{2}=N_{1}^{\prime} \times_{f}\{0\} \oplus N_{1} \times_{f} M_{2}$.

Corollary 2.6. (1) If $f$ is zero, then $M_{1} \times_{f} M_{2}$ is a decomposable $R[X]$-module.

(2) If $\operatorname{Im}(f)$ is a direct summand of $M_{1}$, then $\operatorname{Im}(f) \times_{f} M_{2}$ is a direct summand of $M_{1} \times_{f} M_{2}$.

Proof. Obvious.

\section{Isomorphism Classes}

Let $R$ be a commutative ring. Let $M_{n, m}(R), M_{n}(R)$, and $G l_{n}(R)$ denote, respectively, the set of the $n \times m$ matrices, the set of the $n \times n$ matrices, and the set of the $n \times n$ invertible matrices, with entries in $R$, where $m$ and $n$ are two nonzero natural numbers.

Let $M_{1}$ and $M_{2}$ be two $R[X]$-modules that are $R$-free of respective finite rank $n$ and $m$. We assume that $M_{1}=R^{n}$ and $M_{2}=R^{m}$ (as $R$-modules). Let $\mathbf{B}_{1}$ and $\mathbf{B}_{2}$ be the respective canonical bases of $M_{1}$ and $M_{2}$. Let $f, g \in \operatorname{Hom}_{R}\left(M_{2}, M_{1}\right)$, let $C_{f}=M_{a t} \mathbf{B}_{2}, \mathbf{B}_{1}(f)$ and 
$C_{g}=\operatorname{Mat}_{\mathbf{B}_{2}, \mathbf{B}_{1}}(g)$, and let $A$ (respectively, $B$ ) be the matrix representing the action of $X$ on $M_{1}$ with respect to the base $\mathbf{B}_{1}$ (respectively, $M_{2}$ with respect to the base $\mathbf{B}_{2}$ ).

Remark 3.1. (1) Let $\widetilde{\mathbf{B}}$ be the canonical base of $M_{1} \times_{f} M_{2}$ seen as $R$-module. Then, the matrix representing the action of $X$ on $M_{1} \times_{f} M_{2}$ with respect to the base $\widetilde{\mathbf{B}}$ is $\left(\begin{array}{ll}A & C_{f} \\ 0 & B\end{array}\right)$.

(2) The modules $M_{1} \times_{f} M_{2}$ and $M_{1} \times_{g} M_{2}$ are isomorphic if and only if $\left(\begin{array}{cc}A & C_{f} \\ 0 & B\end{array}\right)$ and $\left(\begin{array}{cc}A & C_{g} \\ 0 & B\end{array}\right)$ are similar.

The study of isomorphism classes of modules $M_{1} \times_{f} M_{2}$ led us to introduce an equivalence relation on the matrices that is stronger than that of the similarity, and that is the object of the following definition.

Definition 3.2. Let $A, A^{\prime} \in M_{n}(R), B, B^{\prime} \in M_{m}(R)$, and $C, C^{\prime} \in M_{n, m}(R)$, where $n$ and $m$ are two nonzero natural numbers. The matrices $\left(\begin{array}{ll}A & C \\ 0 & B\end{array}\right)$ and $\left(\begin{array}{ll}A^{\prime} & C^{\prime} \\ 0 & B^{\prime}\end{array}\right)$ are called strongly similar if there exists $(U, V) \in G l_{n}(R) \times G l_{m}(R)$ and $T \in M_{n, m}(R)$ such that

$$
\left(\begin{array}{cc}
U & T \\
0 & V
\end{array}\right)\left(\begin{array}{ll}
A & C \\
0 & B
\end{array}\right)=\left(\begin{array}{ll}
A^{\prime} & C^{\prime} \\
0 & B^{\prime}
\end{array}\right)\left(\begin{array}{ll}
U & T \\
0 & V
\end{array}\right) .
$$

For two matrices $M$ and $M^{\prime}$, we put $M \widetilde{s} M^{\prime}$ (respectively, $M \widetilde{\widetilde{s}} M^{\prime}$ ) to mean that $M$ and $M^{\prime}$ are similar (respectively, strongly similar).

Remark 3.3. For $U, V \in M_{n}(R) \times M_{m}(R)$ and $T \in M_{n, m}(R)$, it is easy to see that $\left(\begin{array}{cc}U & T \\ 0 & V\end{array}\right)$ is invertible if and only if $U$ and $V$ are invertible. 
Definition 3.4. Let $f, g \in \operatorname{Hom}_{R}\left(M_{2}, M_{1}\right) . M_{1} \times_{f} M_{2}$ and $M_{1} \times_{g} M_{2}$ are called globally isomorphic, if there exists an isomorphism of $R[X]$-modules $\varphi: M_{1} \times_{f} M_{2} \rightarrow M_{1} \times_{g} M_{2}$, such that $\varphi\left(M_{1} \times\{0\}\right) \subset M_{1} \times\{0\}$.

We have the following interesting result:

Proposition 3.5. The modules $M_{1} \times_{f} M_{2}$ and $M_{1} \times_{g} M_{2}$ are globally isomorphic if and only if $\left(\begin{array}{cc}A & C_{f} \\ 0 & B\end{array}\right)$ and $\left(\begin{array}{cc}A & C_{g} \\ 0 & B\end{array}\right)$ are strongly similar.

Proof. We denote by $\tilde{X}_{f}$ (respectively, $\tilde{X}_{g}$ ) the endomorphism which represents the action of $X$ on $M_{1} \times_{f} M_{2}$ (respectively, $M_{1} \times_{g} M_{2}$ ). The matrix of $\tilde{X}_{f}$ (respectively, $\tilde{X}_{g}$ ) in the canonical base of $M_{1} \times_{f} M_{2}$ (respectively, $M_{1} \times_{g} M_{2}$ ) is $\left(\begin{array}{cc}A & C_{f} \\ 0 & B\end{array}\right)$ (respectively, $\left(\begin{array}{cc}A & C_{g} \\ 0 & B\end{array}\right)$

Assume that $M_{1} \times_{f} M_{2}$ and $M_{1} \times_{g} M_{2}$ are globally isomorphic by an isomorphism $\varphi$. In the canonical bases of $M_{1} \times_{f} M_{2}$ and $M_{1} \times_{g} M_{2}$, $\varphi$ admits a matrix of the form $\left(\begin{array}{cc}U & T \\ 0 & V\end{array}\right)$, where $(U, V) \in G l_{n}(R) \times$ $G l_{m}(R)$ and $T \in M_{n, m}(R)$. As for all $m \in M_{1} \times_{f} M_{2}, \varphi(X . m)=X . \varphi(m)$, then $\varphi \circ \tilde{X}_{f}=\tilde{X}_{g} o \varphi$. So, $\left(\begin{array}{cc}U & T \\ 0 & V\end{array}\right)\left(\begin{array}{cc}A & C_{f} \\ 0 & B\end{array}\right)=\left(\begin{array}{cc}A & C_{g} \\ 0 & B\end{array}\right)\left(\begin{array}{cc}U & T \\ 0 & V\end{array}\right)$. Therefore, $\left(\begin{array}{ll}A & C_{f} \\ 0 & B\end{array}\right)$ and $\left(\begin{array}{cc}A & C_{g} \\ 0 & B\end{array}\right)$ are strongly similar. 
Reciprocally, assume that $\left(\begin{array}{cc}A & C_{f} \\ 0 & B\end{array}\right)$ and $\left(\begin{array}{cc}A & C_{g} \\ 0 & B\end{array}\right)$ are strongly similar. So, there exist $U, V \in G l_{n}(R) \times G l_{m}(R)$ and $T \in M_{n, m}(R)$, such that $\left(\begin{array}{cc}U & T \\ 0 & V\end{array}\right)\left(\begin{array}{cc}A & C_{f} \\ 0 & B\end{array}\right)=\left(\begin{array}{cc}A & C_{g} \\ 0 & B\end{array}\right)\left(\begin{array}{cc}U & T \\ 0 & V\end{array}\right)$. Let $\varphi: M_{1} \times_{f} M_{2}$ $\rightarrow M_{1} \times{ }_{g} M_{2}$ be the morphism of $R$-modules, which is represented by the matrix $\left(\begin{array}{cc}U & T \\ 0 & V\end{array}\right)$. Then, we have $\varphi \circ \widetilde{X}_{f}=\tilde{X}_{g} o \varphi$. So, for all $m \in M_{1}$ ${ }^{\circ} M_{2}, \varphi(X . m)=X . \varphi(m)$. As $\varphi$ is obviously bijective and $\varphi\left(M_{1} \times\{0\}\right) \subset$ $M_{1} \times\{0\}$, then $M_{1} \times_{f} M_{2}$ and $M_{1} \times_{g} M_{2}$ are globally isomorphic.

It is clear that if $M_{1} \times_{f} M_{2}$ and $M_{1} \times_{g} M_{2}$ are globally isomorphic, then they are isomorphic. In the following, we show the equivalence in special cases.

Proposition 3.6. The following conditions are equivalent:

(1) The modules $M_{1} \times_{f} M_{2}$ and $M_{1} \oplus M_{2}$ are globally isomorphic.

(2) The modules $M_{1} \times_{f} M_{2}$ and $M_{1} \oplus M_{2}$ are isomorphic.

(3) There exists $T \in M_{n, m}(R)$ such that $A T-T B=C_{f}$.

(4) $\overline{\left(i_{1}, M_{1} \times_{f} M_{2}, p_{2}\right)}=\overline{\left(i_{1}, M_{1} \oplus M_{2}, p_{2}\right)}$.

Proof. (3) $\Leftrightarrow$ (4): Comes from Corollary 2.4. Then, it suffices to show that $(1) \Leftrightarrow(2) \Leftrightarrow(3)$.

(1) $\Rightarrow(2)$ : Evident.

(2) $\Rightarrow$ (3): Let $\varphi: M_{1} \times_{f} M_{2} \rightarrow M_{1} \oplus M_{2}$ be an isomorphism of $R[X]$-modules. Then, we have $\varphi \circ \tilde{X}=\tilde{X} o \varphi$. This implies that $\left(\begin{array}{cc}A & C_{f} \\ 0 & B\end{array}\right)$ and $\left(\begin{array}{ll}A & 0 \\ 0 & B\end{array}\right)$ are similar. By [5] or [6], the equation $A X-X B=C_{f}$ has a solution in $M_{n, m}(R)$. 
(3) $\Rightarrow$ (1): By Corollary 2.4 and Remark 2.3,

$$
\begin{aligned}
\varphi: M_{1} \times_{f} M_{2} & \rightarrow M_{1} \oplus M_{2} \\
\left(m_{1}, m_{2}\right) & \mapsto\left(m_{1}+T m_{2}, m_{2}\right)
\end{aligned}
$$

is an isomorphism of $R[X]$-modules leaving $M_{1}$ globally invariant.

Remark 3.7. If we assume that the conditions of Proposition 3.6 are satisfied, then

$$
\begin{aligned}
\varphi: M_{1} \times_{f} M_{2} & \rightarrow M_{1} \oplus M_{2} \\
\left(m_{1}, m_{2}\right) & \mapsto\left(m_{1}+T m_{2}, m_{2}\right)
\end{aligned}
$$

is an isomorphism of $R[X]$-modules leaving $M_{1}$ globally invariant. Its reciprocal isomorphism is defined by

$$
\begin{gathered}
\varphi^{-1}: M_{1} \oplus M_{2} \rightarrow M_{1} \times_{f} M_{2} \\
\left(m_{1}, m_{2}\right) \mapsto\left(m_{1}-T m_{2}, m_{2}\right) .
\end{gathered}
$$

Proposition 3.8. If one of the following conditions is true:

(i) The equation $B X-X A=0$ admits 0 as a unique solution.

(ii) There existe $\alpha \in R$ such that $A=\alpha I_{n}$ and $C_{g}$ is invertible.

(iii) There existe $\beta \in R$ such that $B=\beta I_{m}$ and $C_{f}$ is invertible, then the following assertions are equivalent:

(1) $M_{1} \times_{f} M_{2}$ and $M_{1} \times_{g} M_{2}$ are isomorphic.

(2) $M_{1} \times_{f} M_{2}$ and $M_{1} \times_{g} M_{2}$ are globally isomorphic. 
Proof. (1) $\Rightarrow$ (2): Assume that $M_{1} \times_{f} M_{2}$ and $M_{1} \times_{g} M_{2}$ are isomorphic by an isomorphism $\varphi$. Then, by (2) of Remark 3.1, there exist $(U, V) \in M_{n}(R) \times M_{n}(R), T \in M_{n, m}(R)$, and $R \in M_{m, n}(R)$ such that $\left(\begin{array}{ll}U & R \\ T & V\end{array}\right)$ is invertible, and

$$
\left(\begin{array}{cc}
U & R \\
T & V
\end{array}\right)\left(\begin{array}{ll}
A & C_{f} \\
0 & B
\end{array}\right)=\left(\begin{array}{cc}
A & C_{g} \\
0 & B
\end{array}\right)\left(\begin{array}{ll}
U & R \\
T & V
\end{array}\right) .
$$

But,

$$
\left(\begin{array}{cc}
U & R \\
T & V
\end{array}\right)\left(\begin{array}{cc}
A & C_{f} \\
0 & B
\end{array}\right)=\left(\begin{array}{cc}
A & C_{g} \\
0 & B
\end{array}\right)\left(\begin{array}{ll}
U & R \\
T & V
\end{array}\right) \Leftrightarrow\left\{\begin{array}{l}
T A=B T ; \\
U A=A U+C_{g} T ; \\
T C_{f}+V B=B V ; \\
U C_{f}+R B=A R+C_{g} V .
\end{array}\right.
$$

If one of the conditions (i), (ii) or (iii) is true, then $T=0$. Therefore, $\left(\begin{array}{cc}A & C_{f} \\ 0 & B\end{array}\right) \approx\left(\begin{array}{cc}A & C_{g} \\ 0 & B\end{array}\right)$. At last, by Proposition 3.5, $M_{1} \times_{f} M_{2}$ and $M_{1} \times_{g} M_{2}$ are globally isomorphic.

(2) $\Rightarrow(1)$ : Obvious.

Remark 3.9. (1) If $m=n$ and there exists $\alpha \in R$ such that $A=\alpha I_{n}$ and $B-\alpha I_{n}$ is invertible or there exists $\beta \in R$ such that $B=\beta I_{n}$ and $A-\beta I_{n}$ is invertible, then the condition (i) in Proposition 3.8 is satisfied.

(2) In a field the equation $B X-X A=0$ admits 0 as a unique solution means that $A$ and $B$ have no eigenvalue in common. 
Lemma 3.10. Let $\left(m_{1}, m_{2}\right) \in M_{1} \times_{f} M_{2}$ and $n$ be a nonzero natural number. Then,

$$
X^{n} .\left(m_{1}, m_{2}\right)=\left(X^{n} \cdot m_{1}+\sum_{k=0}^{n-1} X^{n-1-k} f\left(X^{k} \cdot m_{2}\right), X^{n} \cdot m_{2}\right) .
$$

Proof. We use an induction on $n$. Let $\left(m_{1}, m_{2}\right) \in M_{1} \times_{f} M_{2}$. For $n=1$, we have

$$
\begin{aligned}
X .\left(m_{1}, m_{2}\right) & =\left(X . m_{1}+f\left(m_{2}\right), X . m_{2}\right) \\
& =\left(X^{1} . m_{1}+\sum_{k=0}^{1-1} X^{1-1-k} f\left(X^{k} . m_{2}\right), X^{1} . m_{2}\right) .
\end{aligned}
$$

Assume that

$$
X^{n} .\left(m_{1}, m_{2}\right)=\left(X^{n} . m_{1}+\sum_{k=0}^{n-1} X^{n-1-k} f\left(X^{k} . m_{2}\right), X^{n} . m_{2}\right) .
$$

Then,

$$
\begin{aligned}
X^{n+1} .\left(m_{1}, m_{2}\right) & =X\left(X^{n} . m_{1}+\sum_{k=0}^{n-1} X^{n-1-k} f\left(X^{k} . m_{2}\right), X^{n} . m_{2}\right) \\
& =\left(X^{n+1} . m_{1}+\sum_{k=0}^{n-1} X^{n-k} f\left(X^{k} . m_{2}\right)+f\left(X^{n} . m_{2}\right), X^{n+1} . m_{2}\right) \\
& =\left(X^{n+1} . m_{1}+\sum_{k=0}^{n} X^{n-k} f\left(X^{k} . m_{2}\right), X^{n+1} \cdot m_{2}\right)
\end{aligned}
$$

Proposition 3.11. Let $\varphi: M_{1} \times_{f} M_{2} \rightarrow M_{1} \times_{g} M_{2}$ be a homomorphism of $R[X]$-modules. If $g$ is an injective homomorphism of $R[X]$-modules, and there exists a nonzero natural number $l$ such that $l$ is invertible in $R$ and both $M_{1}$ and $M_{2}$ are annihilated by $X^{l}-1$, then $\varphi\left(M_{1} \times\{0\}\right) \subset M_{1} \times\{0\}$. 
In particular, if furthermore $\varphi$ is an isomorphism, then $M_{1} \times_{f} M_{2}$ and $M_{1} \times_{g} M_{2}$ are globally isomorphic by the isomorphism $\varphi$.

Proof. Let $\left(m_{1}, 0\right) \in M_{1} \times\{0\}$. We put $\varphi\left(m_{1}, 0\right)=\left(m_{1}^{\prime}, m_{2}^{\prime}\right)$. Then, we have

$$
\begin{aligned}
\varphi\left(m_{1}, 0\right) & =\varphi\left(X^{l} . m_{1}, 0\right) \\
& =\left(X^{l} \cdot m_{1}^{\prime}+\sum_{k=0}^{l-1} X^{l-1-k} g\left(X^{k} . m_{2}^{\prime}\right), X^{l} . m_{2}^{\prime}\right)(\text { By Lemma 3.10) } \\
& =\left(m_{1}^{\prime}, m_{2}^{\prime}\right) .
\end{aligned}
$$

So,

$$
\begin{aligned}
& \sum_{k=0}^{l-1} X^{l-1-k} g\left(X^{k} . m_{2}^{\prime}\right)=0 . \\
\Rightarrow & \left.\sum_{k=0}^{l-1} g\left(X^{l-1} . m_{2}^{\prime}\right)=0 . \text { (Since } g \text { is a homomorphism of } R[X] \text {-modules }\right) \\
\Rightarrow & l g\left(X^{l-1} \cdot m_{2}^{\prime}\right)=0 . \\
\Rightarrow & g\left(X^{l-1} \cdot m_{2}^{\prime}\right)=0 .(\text { Since } l \text { is invertible in } R) \\
\Rightarrow & X^{l-1} \cdot m_{2}^{\prime}=0 . \quad \text { (Since } g \text { is injective) } \\
\Rightarrow & X . X^{l-1} \cdot m_{2}^{\prime}=X^{l} . m_{2}^{\prime}=m_{2}^{\prime}=0 .
\end{aligned}
$$

So, $\varphi\left(m_{1}, 0\right)=\left(m_{1}^{\prime}, 0\right)$. Therefore, $\varphi\left(M_{1} \times\{0\}\right) \subset M_{1} \times\{0\}$.

Corollary 3.12. Let $A \in M_{n}(R), B \in M_{m}(R)$ and $C, C^{\prime} \in M_{n, m}(R)$, where $n$ and $m$ are two nonzero natural numbers. Let $(U, V) \in M_{n}(R) \times$ $M_{m}(R), R \in M_{n, m}(R) \quad$ and $\quad T \in M_{m, n}(R) \quad$ such that $\left(\begin{array}{ll}U & R \\ T & V\end{array}\right)$ 
$\left(\begin{array}{cc}A & C \\ 0 & B\end{array}\right)=\left(\begin{array}{ll}A & C^{\prime} \\ 0 & B\end{array}\right)\left(\begin{array}{ll}U & R \\ T & V\end{array}\right)$. If $C^{\prime}$ is invertible, $A C=C^{\prime} B$ and there exists a nonzero natural number $l$ such that $l$ is invertible in $R$, $A^{l}=I_{n}$ and $B^{l}=I_{m}$, then $T=0$.

In particular, if $\left(\begin{array}{cc}A & C \\ 0 & B\end{array}\right) \widetilde{s}\left(\begin{array}{cc}A & C^{\prime} \\ 0 & B\end{array}\right)$, then $\left(\begin{array}{cc}A & C_{f} \\ 0 & B\end{array}\right) \approx$ $\left(\begin{array}{cc}A & C_{g} \\ 0 & B\end{array}\right)$

Proof. Just take $M_{1}=R^{n}, M_{2}=R^{m}, \mathbf{B}_{1}$ and $\mathbf{B}_{2}$ the respective canonical bases of $M_{1}$ and $M_{2}, C=C_{f}=M a t_{\mathbf{B}_{2}, \mathbf{B}_{1}}(f), C^{\prime}=C_{g}=$ $M a t_{\mathbf{B}_{2}, \mathbf{B}_{1}}(g)$ and $A$ (respectively, $B$ ) the matrix representing the action of $X$ on $M_{1}$ with respect to the base $\mathbf{B}_{1}$ (respectively, $M_{2}$ with respect to the base $\mathbf{B}_{2}$ ).

Lemma 3.13. If $(U, V) \in M_{n}(R) \times M_{m}(R), R \in M_{n, m}(R), T \in M_{m, n}(R)$

$$
\begin{aligned}
& \text { and }\left(\begin{array}{cc}
U & R \\
T & V
\end{array}\right)\left(\begin{array}{ll}
A & C_{f} \\
0 & B
\end{array}\right)=\left(\begin{array}{cc}
A & C_{g} \\
0 & B
\end{array}\right)\left(\begin{array}{cc}
U & R \\
T & V
\end{array}\right) \text {, then }\left(\begin{array}{cc}
A & U C_{f} \\
0 & B
\end{array}\right) \\
& \widetilde{\widetilde{s}}\left(\begin{array}{cc}
A & C_{g} V \\
0 & B
\end{array}\right) .
\end{aligned}
$$

Proof.

$$
\begin{gathered}
\left(\begin{array}{cc}
U & R \\
T & V
\end{array}\right)\left(\begin{array}{ll}
A & C_{f} \\
0 & B
\end{array}\right)=\left(\begin{array}{cc}
A & C_{g} \\
0 & B
\end{array}\right)\left(\begin{array}{ll}
U & R \\
T & V
\end{array}\right) \\
\Rightarrow U C_{f}+R B=A R+C_{g} V .
\end{gathered}
$$


Then, we have

$$
\begin{gathered}
\left(\begin{array}{cc}
I_{n} & R \\
0 & I_{m}
\end{array}\right)\left(\begin{array}{cc}
A & U C_{f} \\
0 & B
\end{array}\right)\left(\begin{array}{cc}
I_{n} & -R \\
0 & I_{m}
\end{array}\right)=\left(\begin{array}{cc}
A & C_{g} V \\
0 & B
\end{array}\right) . \\
\operatorname{As}\left(\begin{array}{cc}
I_{n} & R \\
0 & I_{m}
\end{array}\right)^{-1}=\left(\begin{array}{cc}
I_{n} & -R \\
0 & I_{m}
\end{array}\right), \operatorname{then}\left(\begin{array}{cc}
A & U C_{f} \\
0 & B
\end{array}\right) \approx\left(\begin{array}{cc}
A & C_{g} V \\
0 & B
\end{array}\right) .
\end{gathered}
$$

Proposition 3.14. Assume that $M_{1} \times_{f} M_{2}$ and $M_{1} \times_{g} M_{2}$ are isomorphic by an isomorphism $\varphi$. Let $f^{\prime}=p_{1} \circ \varphi \circ i_{1}$ of and $g^{\prime}=g_{0} p_{2} \circ o_{0} i_{2}$. Then, $M_{1} \times_{f^{\prime}} M_{2}$ and $M_{1} \times_{g^{\prime}} M_{2}$ are globally isomorphic.

Proof. Comes from Lemma 3.13.

Remark 3.15. In general, $M_{1} \times_{f} M_{2}$ and $M_{1} \times_{g} M_{2}$ are globally isomorphic does not necessarily imply that $\overline{\left(i_{1}, M_{1} \times_{f} M_{2}, p_{2}\right)}=$ $\overline{\left(i_{1}, M_{1} \times_{g} M_{2}, p_{2}\right)}$. In particular, $M_{1} \times_{f} M_{2}$ and $M_{1} \times_{g} M_{2}$ are isomorphic does not necessarily imply that $\overline{\left(i_{1}, M_{1} \times_{f} M_{2}, p_{2}\right)}=$ $\overline{\left(i_{1}, M_{1} \times_{g} M_{2}, p_{2}\right)}$. Indeed, let $R=\mathbb{R}$ and let $\left(\begin{array}{ll}2 & 3 \\ & \\ 0 & 2\end{array}\right)$ (respectively, $\left(\begin{array}{ll}2 & 1 \\ 0 & 2\end{array}\right)$ ) be the matrix representing the action of $X$ on $M_{1} \times_{f} M_{2}$ (respectively, $M_{1} \times_{g} M_{2}$ ) with respect to its canonical base (as $R$-module). We have $\left(\begin{array}{ll}2 & 3 \\ 0 & 2\end{array}\right) \widetilde{\widetilde{s}}\left(\begin{array}{ll}2 & 1 \\ 0 & 2\end{array}\right)$. So, by Proposition 3.5, $M_{1} \times_{f} M_{2}$ and $M_{1} \times_{g} M_{2}$ are globally isomorphic. But, the equation $2 \times X-X \times 2=3-1$ has not any solution. By Corollary 2.4, $\overline{\left(i_{1}, M_{1} \times_{f} M_{2}, p_{2}\right)} \neq \overline{\left(i_{1}, M_{1} \times_{g} M_{2}, p_{2}\right)}$. 
Lemma 3.16. If $\left(\begin{array}{ll}a & c \\ 0 & b\end{array}\right),\left(\begin{array}{ll}a & c^{\prime} \\ 0 & b\end{array}\right) \in M_{2}(R)$ and $R$ is an integral domain, then

$$
\left(\begin{array}{ll}
a & c \\
0 & b
\end{array}\right) \widetilde{s}\left(\begin{array}{ll}
a & c^{\prime} \\
0 & v
\end{array}\right) \Rightarrow\left(\begin{array}{ll}
a & c \\
0 & b
\end{array}\right) \approx\left(\begin{array}{ll}
a & c^{\prime} \\
0 & v
\end{array}\right) .
$$

Proof. If $\left(\begin{array}{ll}a & c \\ 0 & b\end{array}\right) \tilde{s}\left(\begin{array}{cc}a & c^{\prime} \\ 0 & v\end{array}\right)$, then there exists $\left(\begin{array}{ll}u & r \\ t & v\end{array}\right) \in G l_{2}(R)$

such that

$$
\left(\begin{array}{ll}
u & r \\
t & v
\end{array}\right)\left(\begin{array}{ll}
a & c \\
0 & b
\end{array}\right)=\left(\begin{array}{ll}
a & c^{\prime} \\
0 & b
\end{array}\right)\left(\begin{array}{ll}
u & r \\
t & v
\end{array}\right) .
$$

Or

$$
\begin{gathered}
\left(\begin{array}{cc}
u & r \\
t & v
\end{array}\right)\left(\begin{array}{ll}
a & c \\
0 & b
\end{array}\right)=\left(\begin{array}{ll}
a & c^{\prime} \\
0 & b
\end{array}\right)\left(\begin{array}{ll}
u & r \\
t & v
\end{array}\right) \Leftrightarrow\left\{\begin{array}{l}
t a=b t \\
u a=a u+c^{\prime} t ; \\
t c+v b=b v ; \\
u c+r b=a r+c^{\prime} v .
\end{array}\right. \\
\Rightarrow t=0 \text { or } c=c^{\prime}=0 .
\end{gathered}
$$

It is clear that if $t=0$ or $c=c^{\prime}=0$, then $\left(\begin{array}{ll}a & c \\ 0 & b\end{array}\right) \approx \widetilde{\widetilde{s}}\left(\begin{array}{ll}a & c^{\prime} \\ 0 & b\end{array}\right)$.

If $R$ is an integral domain and $n=m=1$, then we have the following proposition:

Proposition 3.17. The following conditions are equivalent:

(1) $M_{1} \times_{f} M_{2}$ and $M_{1} \times_{g} M_{2}$ are isomorphic.

(2) $M_{1} \times_{f} M_{2}$ and $M_{1} \times_{g} M_{2}$ are globally isomorphic. 
Proof. Clear by (2) of Remark 3.1, Lemma 3.16 and Proposition 3.5.

\section{References}

[1] H. Cartan and S. Eilenberg, Homological Algebra, 7th Edition, Princeton University Press, New Jersey, 1973.

[2] M. E. Charkani and S. Bouhamidi, Modular representation of loewy length two, International Journal of Mathematics and Mathematical Sciences 70 (2003), 4399-4408.

[3] C. W. Curtis and I. Reiner, Methods of Representation Theory, Vol. I, WileyInterscience, New York, 1981.

[4] R. B. Feinberg, Similarity of partitioned matrices, J. Res. Nat. Bur. Standards Sect. B 79B (1975), 117-125.

[5] W. H. Gustafson, Roths theorems over commutative rings, Linear Algebra Appl. 23 (1979), 245-251.

[6] W. H. Gustafson, J. Zelmanowitz, On matrix equivalence and matrix equations, Linear Algebra Appl. 27 (1979), 219-224.

[7] P. J. Hilton and U. Stammbach, A Course in Homological Algebra, Graduate Texts in Mathematics, Vol. 4, Springer-Verlag, Berlin, 1971.

[8] A. Najim and M. E. Charkani, Finitely generated modules over group rings of a direct product of two cyclic groups, Algebra, Vol. 2014, Article ID 256020, 5 pages, 2014. doi:10.1155/2014/256020.

[9] K. Roggenkamp and V. Huber-Dyson, Lattices Over Orders I, Lecture Notes in Mathematics, Vol. 115, Springer-Verlag, Berlin, 1970. 